\title{
Characterization of fluorescent polystyrene microspheres for advanced flow diagnostics
}

\author{
Pietro M.F. Maisto*, K. Todd Lowe ${ }^{\dagger}$, \\ Virginia Tech, Blacksburg, VA, 24061-0203 \\ Gwibo Byun ${ }^{\ddagger}$, Roger Simpson ${ }^{\S}$, \\ AUR, Inc., Blacksburg, VA, 24060 \\ and \\ Max Verkamp ${ }^{* *}$, Jason E. Danley ${ }^{\dagger \dagger}$, Brian Koh ${ }^{\star *}$, Pacita I. Tiemsin, ${ }^{\S \S}$ Paul M. Danehy, ${ }^{* * *}$ Christopher J. Wohl ${ }^{\dagger \dagger}$ \\ NASA Langley Research Center, Hampton, VA
}

\begin{abstract}
Fluorescent dye-doped polystyrene latex microspheres (PSLs) are being developed for velocimetry and scalar measurements in variable property flows. Two organic dyes, Rhodamine B (RhB) and dichlorofluorescein (DCF), are examined to assess laser-induced fluorescence (LIF) properties for flow imaging applications and single-shot temperature measurements. A major interest in the current research is the application of safe dyes, thus DCF is of particular interest, while RhB is used as a benchmark. Success is demonstrated for single-point laser Doppler velocimetry (LDV) and also imaging fluorescence, excited via a continuous wave $2 \mathrm{~W}$ laser beam, for exposures down to $10 \mathrm{~ms}$. In contrast, when exciting with a pulsed Nd:YAG laser at $200 \mathrm{~mJ} /$ pulse, no fluorescence was detected, even when integrating tens of pulses. We show that this is due to saturation of the LIF signal at relatively low excitation intensities, 4-5 orders of magnitude lower than the pulsed laser intensity. A two-band LIF technique is applied in a heated jet, indicating that the technique effectively removes interfering inputs such as particle diameter variation. Temperature measurement uncertainties are estimated based upon the variance measured for the two-band LIF intensity ratio and the achievable dye temperature sensitivity, indicating that particles developed to date may provide about $\pm 12.5{ }^{\circ} \mathrm{C}$ precision, while future improvements in dye temperature sensitivity and signal quality may enable single-shot temperature measurements with sub-degree precision.
\end{abstract}

\footnotetext{
* Graduate Research Assistant, Department of Aerospace and Ocean Engineering, 215 Randolph Hall, AIAA Member

${ }^{\dagger}$ Assistant Professor, Department of Aerospace and Ocean Engineering, 215 Randolph Hall, AIAA Senior Member

* Mechanical Engineer, AIAA Senior Member

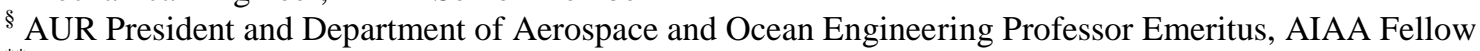

NASA LARSS Student, currently undergraduate at the Chemistry Dept at Rose-Hulman Institute of Technology, Terre Haute, IN

${ }^{\top}$ NASA LARSS Student, currently undergraduate at the Chemistry Dept at University of Nebraska, Lincoln, NE

NASA LARSS Student, currently undergraduate at the Mechanical Engineering Dept at Georgia Institute of Technology, Atlanta, GA

$\$ \S$ Research Scientist, Advanced Sensing and Optical Measurements Branch

${ }^{* * *}$ Research Scientist, Advanced Sensing and Optical Measurement Branch, MS 493, Associate Fellow AIAA.

${ }^{\dagger \dagger}$ Research Chemist, Advanced Materials and Processing Branch
}

1

American Institute of Aeronautics and Astronautics 


\section{Nomenclature}

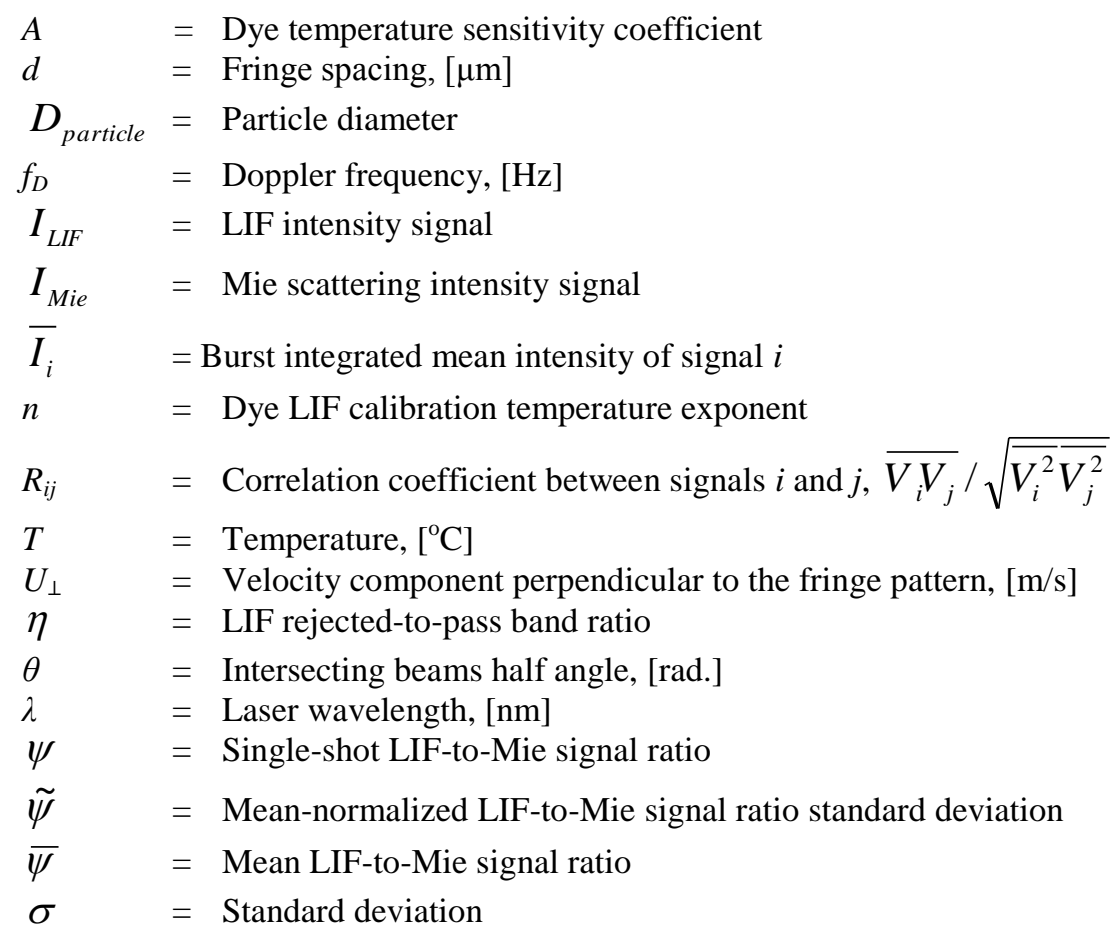

\section{Introduction}

$\mathrm{P}$ olystyrene latex microspheres (PSLs) are a commonly used seed material in laser-based wind tunnel measurement techniques such as laser Doppler velocimetry (LDV) and particle image velocimetry (PIV). PSLs make good seed materials due to their high index of refraction resulting in efficient light scattering as well as their uniform, tunable size distribution obtained via the synthesis process of soap-free emulsion.[1] By adding a fluorescent dye to the reagents, particles may be produced that exhibit laser-induced fluorescence (LIF) in addition to Mie scattering when excited with lasers commonly used for velocimetry. While Mie scattering is at the same wavelength as the incident laser light, fluorescence generally occurs at many longer wavelengths, in addition to the excitation wavelength; leveraging this shift in wavelength can be advantageous near solid surfaces (e.g. walls) since the incident laser light may be filtered to remove flare from the fluorescence signal. An additional interesting application is scalar mixing in multiple flow streams: seeding the streams by means of particles doped with different dyes (i.e. different fluorescence spectra) enables scalar sensing (e.g. concentration and temperature) and vector velocity of the streams.

Several authors have described efforts to dope particles, both liquid and solid, with fluorescent dyes and luminescent paints to measure flow properties. Stevenson et al. reported an early application using the fluorescence signal from dye-doped liquid particles for velocimetry.[3] A common application in liquids - and to a lesser extent, liquid-doped particles-is thermometry. [4, 5] In the current application, liquid particle techniques such as fog-based seeding are problematic in the target large wind tunnel facilities because of the large resulting particle diameters $(>1 \mu \mathrm{m})$ and large particle size distributions. Solid thermographic phosphor particles have also been investigated for their potential in simultaneous temperature and velocity imaging. However, such particles, typically containing rare-earth elements such as Europium, are not suitable for large flow facilities because of health risks, and the abrasive nature of many of these seed materials can be detrimental to the model surface and facility walls. Dye-doped PSLs have been fabricated, studied and applied for a variety of applications, including PIV, for more than three decades. [8-10] PSLs doped with fluorescent dyes are available commercially but are prohibitively expensive for large-scale wind tunnel tests, as is the focus of the current effort.

As an example of the application of doped particles, consider further the laser Doppler velocimetry technique. The simplest form of LDV measures one component of the velocity of the flow at one point in a flow. A laser beam is split and the two paths intersect developing an interference pattern within a small measurement volume. When a seed particle passes through the interference pattern, the particle scatters laser light, which is 
detected via collection optics placed outside of the flow. The magnitude of the component of velocity perpendicular to the spacing of the interference fringe pattern,

$$
U_{\perp}=f_{D} d
$$

can be determined from the measured frequency of the scattered light given the fringe spacing,

$$
d=\frac{\lambda}{2 \sin \theta}
$$

As may be noted, the fringe spacing equation is a unique function of the laser wavelength used and the beam geometry of the probe. As such, the terms in Eq. (2) may be measured precisely for any given instrument setup.

A fluorescent particle still provides elastic Mie scattering at the excitation wavelength as commonly acquired for LDV, but it will also produce a signal from the fluorescence that can be readily separated from the Miescattered signal using optical filters. The time variation of the intensity from the fluorescence signal closely follows the time variation of the local laser fluence on the particle due to the short fluorescence lifetime (ns to $\mu \mathrm{s})$. As such, the fluorescence signal may be used in place of the Mie-scattered signal to obtain particle velocity, as demonstrated previously by the authors for doped PSLs [2], in addition to scalar information about the particle.

Of particular interest in the current effort is the development of dye-doped particles which mitigate health risks to those working around wind tunnels seeded with these particles. Much of the effort reported herein concentrates on the characterization of particles doped with dichlorofluorescein (DCF), a dye used in medical assays and exhibiting low toxicity.

\section{Experimental Setup}

\section{A. Particle synthesis and characterization}

The polystyrene latex microspheres were synthesized using dispersion polymerization in aqueous solutions. In a typical reaction, styrene monomer $(200 \mathrm{~g}, 1.9 \mathrm{~mol})$, fluorescent dye $(0.01-0.1 \mathrm{mg})$, and ionic dispersion stabilizers (typically $\mathrm{MgSO}_{4}$ or $\mathrm{NaHCO}_{3}$ ) were added at millimolar $(\mathrm{mM})$ concentrations to a reaction kettle along with deionized water $(18 \mathrm{M} \Omega, 2 \mathrm{~L})$. The reaction mixture was stirred at $150 \mathrm{rpm}$ using a mechanical stirrer and sparged of oxygen using a gas diffuser and a nitrogen stream $(450 \mathrm{sccm})$ for approximately 45 minutes while the solution temperature was raised to $70{ }^{\circ} \mathrm{C}$. Once thermally equilibrated to $70{ }^{\circ} \mathrm{C}$, the gas diffuser was removed and the stir speed was increased to $250 \mathrm{rpm} . \mathrm{K}_{2} \mathrm{~S}_{2} \mathrm{O}_{8}(1.6 \mathrm{~g}, 6 \mathrm{mmol})$, a radical polymerization initiator, was added to the reaction mixture and the solution was stirred overnight under an inert atmosphere $\left(\mathrm{N}_{2}\right)$. The next day, the reaction mixture was poured through cheese cloth to remove the large amorphous polystyrene portions and the filtrate was stored for later use. The filtrate typically contained $9 \%$ solids by weight. Particle size statistics were determined using a 780 AccuSizer by Particle Sizing Systems, Inc. and optical microscopy.

\section{B. Flow instrumentation}

\section{Optical instruments}

The optical arrangement depicted in Fig.1 was designed for LDV-LIF tests at Virginia Tech. A Coherent Verdi V18 (continuous wave, frequency doubled $\mathrm{Nd}: \mathrm{YVO}_{4}$ laser) at $532 \mathrm{~nm}$ wavelength was employed as the excitation light source for the experiments. The LDV probe volume was formed by splitting into two beams and subsequently focusing and crossing the $30 \mu \mathrm{m}$ diameter waists of the beams at the center of a jet of hot gas from a heater-nozzle assembly using a single $75 \mathrm{~mm}$ focal length achromatic lens. The fringe spacing estimated according to Eq.(2) was $1.0 \mu \mathrm{m}$, while the value for $\theta$ was estimated to be $14.9^{\circ}$.

The light collection optical system was situated in proximity to the jet nozzle exit to collect light from the $90^{\circ}$ side scattering direction relative to the LDV probe beams. The two lenses (not pictured in Fig. 1) in the collection system were operated at an infinite conjugate ratio such that the collection lens collimated the signal and the coupling lens focused it into a fiber. This was accomplished by employing a $100 \mathrm{~mm}$ focal length, 50.8 $\mathrm{mm}$ diameter achromatic collection lens and a $75 \mathrm{~mm}$ focal length, $50.8 \mathrm{~mm}$ diameter achromatic fiber coupling lens. The coupling lens focused the collected light into a $62.5 \mu \mathrm{m}$ core diameter, 0.27 numerical aperture multimode fiber. 
The photodetection scheme is illustrated in Fig. 2. Three photomultiplier tubes (PMT) were used to sense Mie (Hamamatsu R4124) and LIF (Hamamatsu R9110 x 2) signals. To separate the fluorescence signal from the Mie signal at $532 \mathrm{~nm}$, the optical signal from the fiber was split using a $532 \mathrm{~nm}$ blocking filter (OD6 with $17 \mathrm{~nm}$ FHWM centered at $533 \mathrm{~nm}$ ). The $532 \mathrm{~nm}$ Mie scattering signal was back reflected and detected with the R4124 PMT. The transmitted signal was then further split using a tunable dichroic filter (Semrock Inc. model TBP01561). At $25^{\circ}$ angle of incidence, as chosen based upon the spectral behavior of the DCF, the passband of this filter was $539 \mathrm{~nm}-559 \mathrm{~nm}$ and the reflected signal consisted of all other detectable wavelengths except the band around $533 \mathrm{~nm}$ already filtered. The two R9110 PMTs were used to measure signals from these bands split by the filter.

Each of the PMTs was terminated across a 50 Ohm impedance at the input of Sonoma Instruments model 315 low noise amplifiers, which delivered $51 \mathrm{~dB}$ of gain. Rather than simply terminating the PMTs with a large impedance, these amplifiers were used in order to preserve the time-response of the signal for LDV measurements. The three PMTs were supplied with independent high voltage power supplies. The Mie/LDV channel PMT was supplied over the range -275.5 to $-375 \mathrm{~V}$, while the LIF PMTs were supplied with -550 to -650 $\mathrm{V}$ for all cases under investigation. The voltage was recorded for each measurement for intensity comparisons among the channels. Data from the amplified PMT signals were acquired using a PC-based digitizer controlled using LabVIEW. The signals were sampled at $250 \mathrm{MSamples} / \mathrm{s}$ and 14 bits vertical resolution in triggered bins of 16,384 samples. All data were then stored to hard disk for offline post-processing.

The post-processing relied upon the electronic trigger set in the experiment in order to detect the presence of a Mie or LIF signal burst arising from a single particle passing through the measurement volume. For each burst detected, several features of the signal were recorded including the offset-corrected mean and root-mean-square burst voltages; the Doppler frequency as it appears in Eq. (1) was determined from both the Mie and LIF channels.

\section{Flow rig}

The setup included a jet flow rig designed to provide air at a controlled temperature and total pressure resulting in jet velocities of nominally $20 \mathrm{~m} / \mathrm{s}$ at the nozzle exhaust. The PSL particles, which were initially suspended as an aqueous colloid, were nebulized inside of a plenum using an ultrasonic humidifier, and a filtered compressed air line supply provided dilution and flow volume. It was estimated that the aerosol mixture has a 2 second residence time within the plenum to allow for adequate flow equilibration and evaporation of the solvent in the atomized PSL mixture. A corrugated flexible tube connected the plenum and jet nozzle. The flow was heated using an Omega Engineering model AHF-12240 120/240 VAC electric heater which can deliver up to 200 CFM of air at temperatures up to $315{ }^{\circ} \mathrm{C}$. The jet outlet temperature was controlled by varying the AC voltage using a variable transformer. The $10.2 \mathrm{~cm}$ inner diameter heater tube was reduced to $14.2 \mathrm{~mm}$ diameter at the outlet via an ad hoc nozzle contraction design, providing an area ratio greater than 7 to minimize the impact of heater element and instrumentation wakes. The flow pressure and temperature were monitored by conventional instrumentation (a K-type thermocouple with an accuracy of $\pm 1^{\circ} \mathrm{C}$ and a plenum pressure port connected to a digital manometer with $0.01 \mathrm{kPa}$ resolution). The two LDV probe beams were oriented to measure purely the velocity component along the jet axis (Fig. 1). 


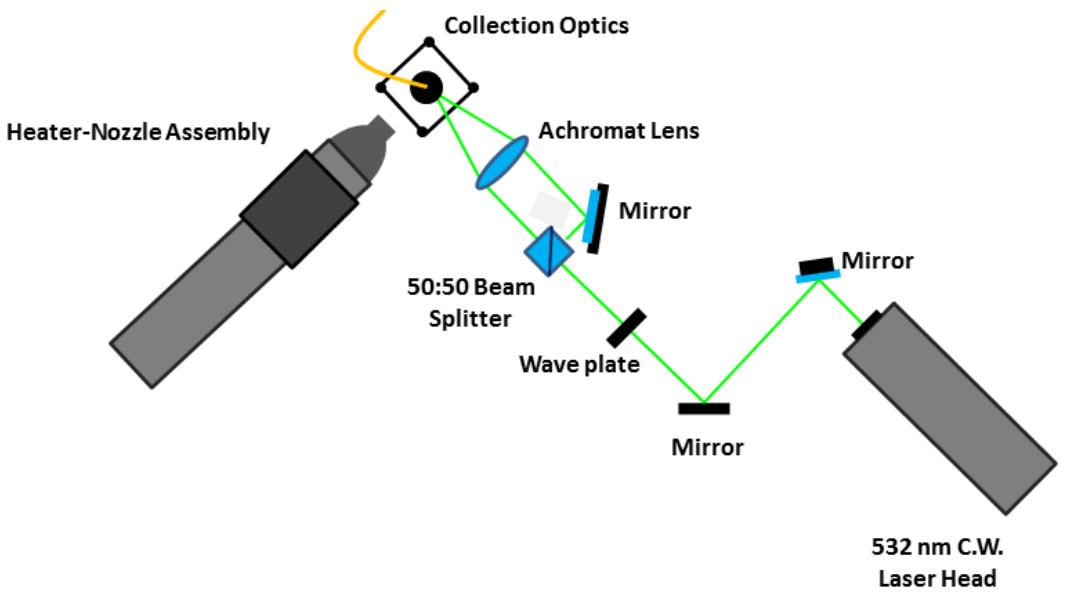

Figure 1. Experimental setup for the bench-top LDV-LIF measurements schematic (top view).

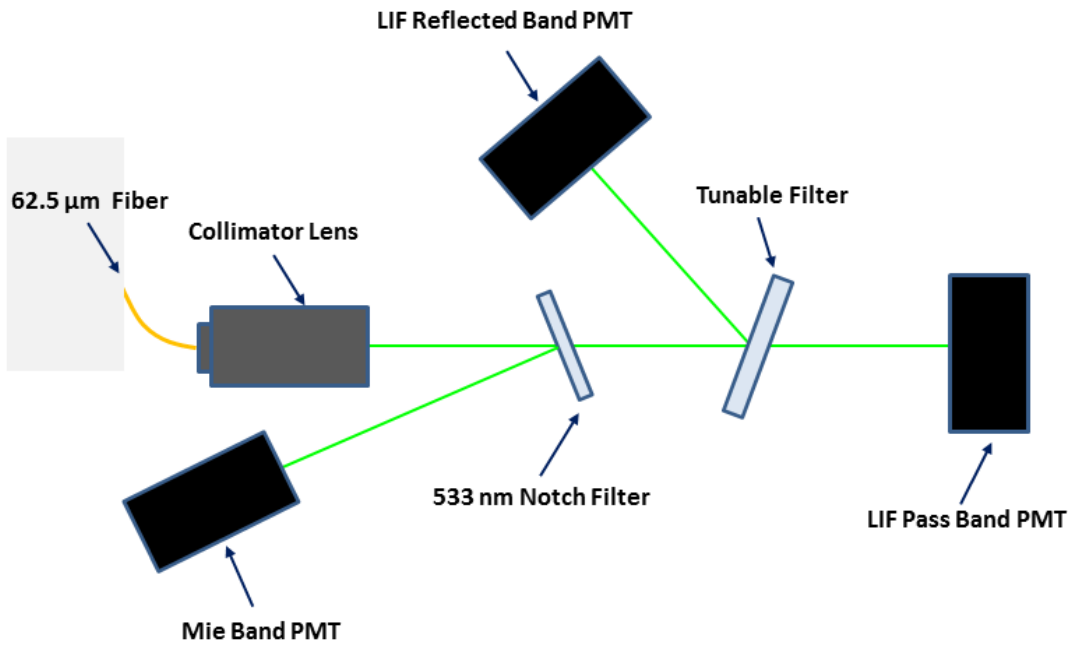

Figure 2. Detector system schematic: Hamamatsu R9110 PMTs collect LIF signal that is transmitted (passband = $539-558.8 \mathrm{~nm}$ FWHM at $25^{\circ}$ angle of incidence and reflected wavelengths $>559 \mathrm{~nm}$ ) by a tunable bandpass filter. A Hamamatsu R4124 PMT collects Mie signal reflected by a $533 \mathrm{~nm}$ notch filter, which was set $12^{\circ}$ counterclockwise with respect to the plane perpendicular to the laser beam; the tunable filter was set $25^{\circ}$ clockwise.

For conducting the particle experiments, particle batches were prepared as follows: $20 \mathrm{~mL}$ of concentrated PSL mixture, approximately $9 \mathrm{wt} \%$ solids, was diluted in $10 \mathrm{~mL}$ distilled water. This mixture was then sonicated in order to eliminate agglomeration using an ultrasonic cleaner. Upon removal from sonication, the mixture was poured immediately into the ultrasonic humidifier reservoir.

3. Particle spectral characterization

Fluorescence spectra were collected for samples of PSLs deposited onto a microscope slide and subsequently dried. This microscope slide was placed on a hot plate and the temperature was increased while exciting the sample using a low-power frequency-doubled Nd:YAG CW excitation source. A $532 \mathrm{~nm}$ blocking filter was used in front of a collection fiber optic connected to an Ocean Optics spectrometer. A thermocouple in close proximity to the illuminated region was used to observe the approximate PSL temperature.

\section{Results}

\section{A. Two-band LIF technique}

Several authors have described the use of two-color or two-band LIF techniques for temperature measurements using organic dyes, e.g. $[4,5]$. The method is employed to remove interfering inputs from 
fluorescence measurements, such as absolute excitation flux and local variations in dye concentration. In order to achieve two-band LIF measurements, the fluorescence emission spectrum of the dye must exhibit temperature sensitivity which varies significantly with wavelength. As an example, consider the spectra measured for a dried sample of PSLs doped using DCF measured at several temperatures (Fig. 3 top). It is important to note that the absolute fluorescence yield for the sample changes significantly through the temperature range, which one may presume could be used for temperature measurements. However, the uncertainty in absolute levels in single-shot measurements reduces the effectiveness of a single-band technique. By normalizing the spectra by the peak intensity, the spectra collapse over significant wavelength regions while exhibiting monotonic variations in other regions (Fig. 3 bottom). This fact is leveraged in a two-band technique using tunable filters to distinguish the spectral regions of varying sensitivity. An example of such an application is shown in Fig. 4 for the same spectra as in Fig. 3, exhibiting two-band ratiometric sensitivity to temperature.
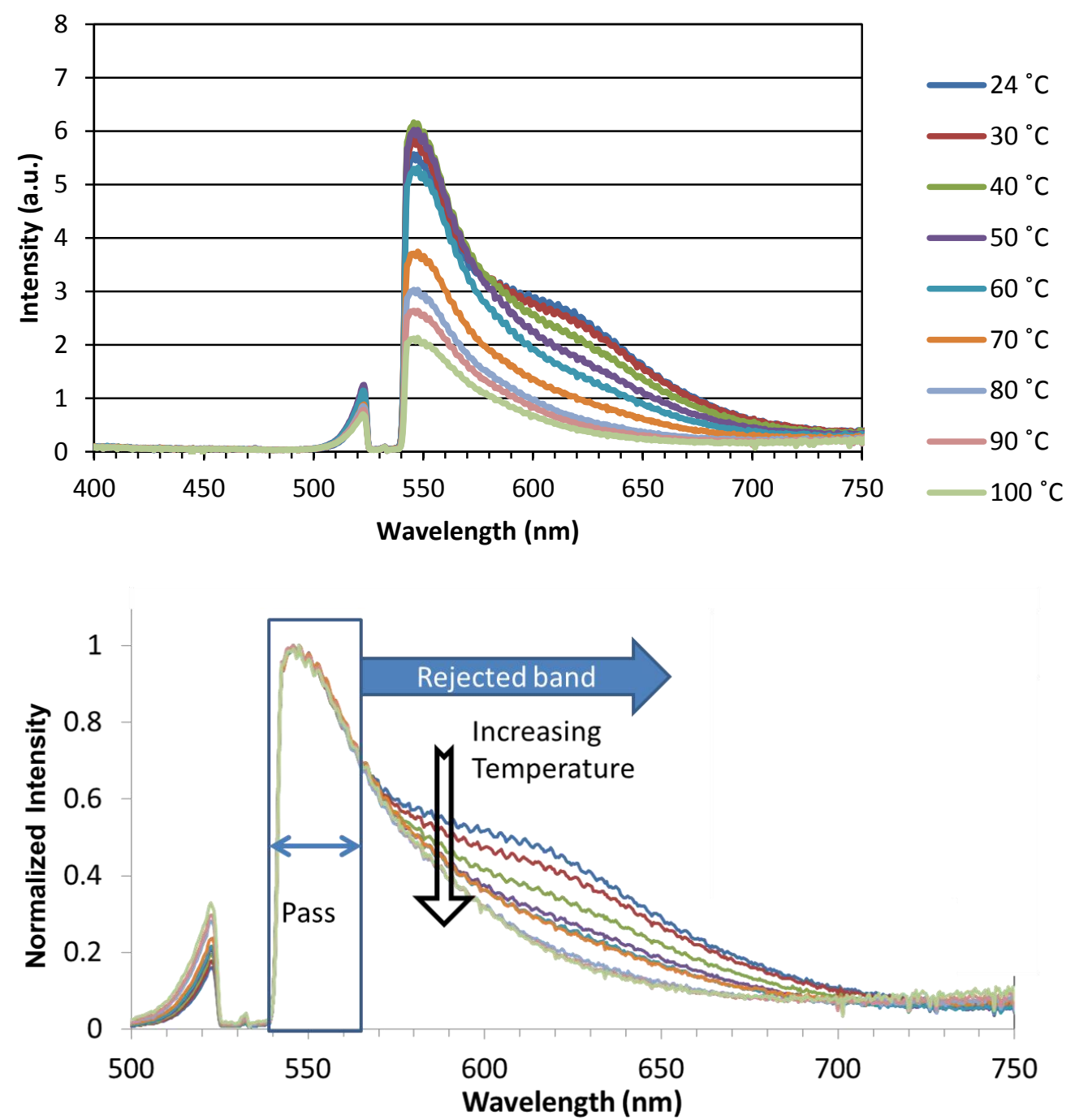

Figure 3. Two-band LIF technique exhibited. Top: raw spectral data acquired for DCF-doped particles. Bottom: Results after normalizing by maximum intensity; Pass/rejected band ratio exhibits temperature sensitivity and immunity from other parameter variations. 


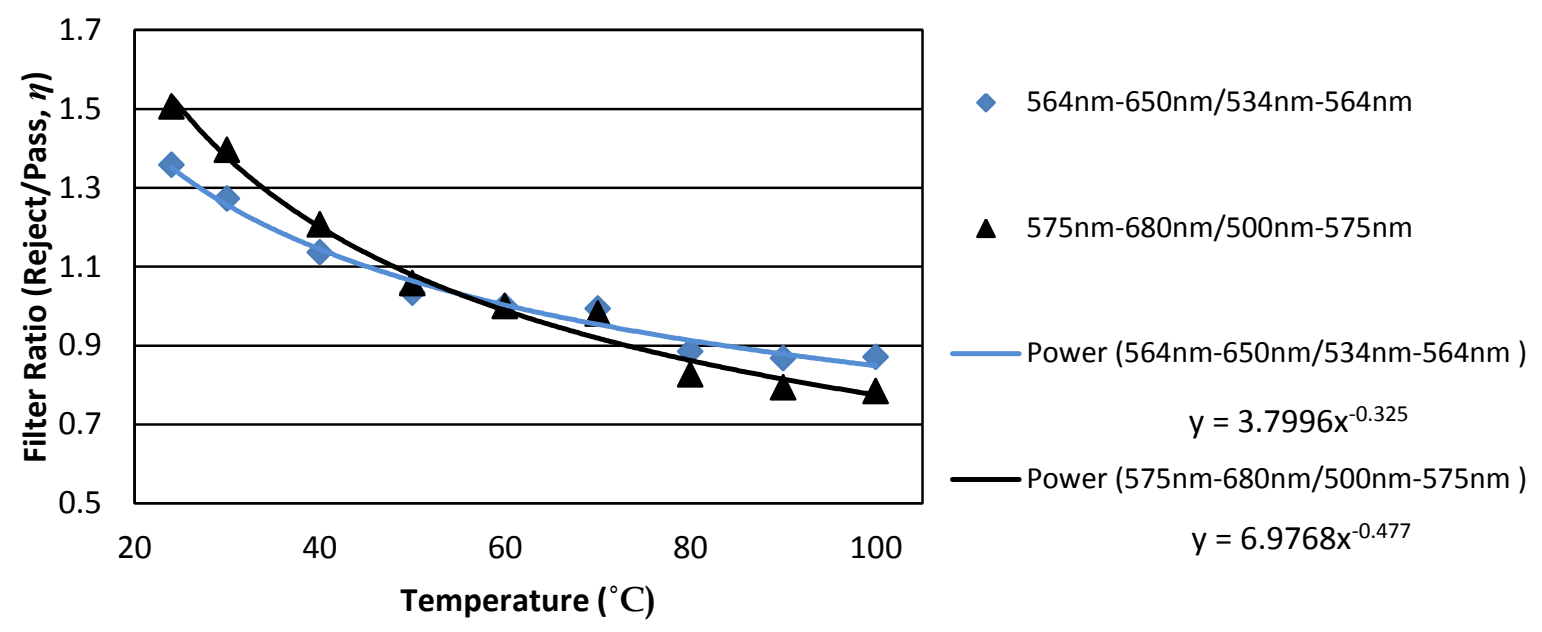

Figure 4. Example of two-band temperature characterization for dried clusters of particles.

\section{A. Single-particle LDV/LIF results}

Measurements were obtained for sets of PSL particles doped with RhB and DCF dyes. Primary particle and dye characteristics of the batches are listed in Table 1 along with diameter statistics. Note that Batches 58 and 60 differ by the addition of a buffer to Batch 60 during the synthesis process in order to produce particles with higher $\mathrm{pH}$ than Batch 58. The excitation laser was set to $4.0 \mathrm{~W}$ or $6.5 \mathrm{~W}$ for most of the runs reported. For DCFBatch 58 (internal designation), the fluorescence was also tested for a sweep of excitation power ranging from 1 to $10 \mathrm{~W}$. All cases were tested for similar flow conditions at the nozzle exhaust with plenum gage pressure of approximately $0.04 \mathrm{kPa}$.

Table 1. Particle characteristics for batches tested in the flow apparatus.

\begin{tabular}{|c|c|c|c|}
\hline Particle/Dye & $\begin{array}{c}\text { Mean Particle } \\
\text { Diameter }[\boldsymbol{\mu m}]\end{array}$ & $\begin{array}{c}\text { Standard Deviation of } \\
\text { Particle Diameters } \sigma[\mu \mathrm{m}]\end{array}$ & Concentration (mmol/L Styrene) \\
\hline RhB-(Designation: Batch 57) & 1.09 & 0.41 & 4.50 \\
\hline DCF-(Designation: Batch 58) & 0.77 & 0.15 & 9.29 \\
\hline $\begin{array}{c}\text { DCF+NaHCO } \text { (Designation: } \\
\text { Batch 60) }\end{array}$ & 1.231 & 1.103 & 1.15 \\
\hline
\end{tabular}

\section{Past work: LDV using LIF signals}

In past work, the authors demonstrated DCF and RhB-doped particles for fluorescence-based velocimetry. [2] Results indicated that the fluorescence signal from RhB-doped particles may be used nearly as well as the Mie signal for obtaining Doppler frequency from LDV bursts (e.g., 95\% of valid Mie scattering velocimetry signals were accompanied by a fluorescence signal producing a valid velocimetry sample). In contrast, the DCF signals examined yielded considerably lower LIF signal-to-noise ratio than the RhB-doped particles (32\% of the LIF signals produced valid velocimetry samples). The results, particularly for $\mathrm{RhB}$ doping, indicated that fluorescence signals may be used in place of Mie signals for measurements near surfaces with the benefit of filtering interfering flare at the excitation wavelength. The work led to additional applications of the particles, including exploration for imaging and two-band techniques.

\section{Characterization of particles for imaging applications}

Imaging affords global views of flow-field phenomena and, in many cases, simplified instrumentation setup and improved measurement efficiency. Several imaging applications would be considered for the currently studied particles, including fluorescence-based PIV as demonstrated by Dong et al. [10] as well as scalar concentration and flow temperature imaging. 
The key to time-resolved imaging is the ability to excite the fluorescence with nanosecond pulsed lasers in order to produce fluorescence levels detectable with CCD or CMOS sensors. A double-pulsed $200 \mathrm{~mJ}$ per pulse, frequency doubled Nd:YAG laser was used to excite fluorescence in batches 57, 58, and 60 studied herein. Interestingly, no fluorescence was observed on our unintensified CMOS sensor when using a long-pass filter which rejects the $532 \mathrm{~nm}$ excitation, even when using a relatively fast lens $(\mathrm{f} / \#=2.8)$. Further, focusing the pulsed beam to a tight spot and averaging several tens of pulses still yielded no detected fluorescence despite verifying that large numbers of particles were present within each pulsed beam via Mie scattering images. Since fluorescence had been measured previously using a CW laser and PMTs as photodetectors, the same $532 \mathrm{~nm}$ CW laser was used for time integrated imaging in order to verify the camera setup with the long pass filter. Exposing the sensor to allow for only $1 / 10^{\text {th }}$ the laser excitation energy of a single pulse ( $2 \mathrm{~W} \mathrm{CW} \mathrm{x} 10 \mathrm{~ms}=20 \mathrm{~mJ}$ ), fluorescence was detectable in the image of the tightly focused spot. Sample images obtained using the CW laser source and three different exposure times $(50 \mathrm{~ms}, 20 \mathrm{~ms}$, and $10 \mathrm{~ms})$ are shown in Fig. 5.

A likely explanation for the lack of fluorescence despite high laser energy delivered by the pulsed laser is the occurrence of fluorescence saturation in the measurements. A separate study was conducted using the CW laser and PMT detectors in which the excitation laser power was varied while fluorescence was measured. The results of this study are shown in Fig. 6 for DCF batch 58. They indicate that at approximately $4 \mathrm{~W}$ excitation for the setup, fluorescence saturates and remains at the same level even for high excitation power. Excitation power flux (intensity) is a natural parameter which would characterize this saturation, which had a value of $5 \mathrm{~W} / \mathrm{mm}^{2}$ for the experimental setup used to obtain Fig. 6 (i.e., beams crossed within the measurement volume at a diameter of 1 $\mathrm{mm})$. Over the course of a $20 \mu$ s particle transit through the CW laser volume, a total energy flux of $0.1 \mathrm{~mJ} / \mathrm{mm}^{2}$ would be obtained. It can be expected that any energy density above this would provide no increase in fluorescence. For the focused spot, estimated to be $100 \mu \mathrm{m}$ in diameter, and pulsed laser, an energy flux of approximately $6 \mathrm{~J} / \mathrm{mm}^{2}$ was delivered, indicating that only 16 parts per million of the excitation would provide fluorescence. As such, there was no advantage to pulsed excitation for the particles examined. Imaging with a 10 $\mathrm{ms}$ or longer exposure time for the current particles still provides opportunities for rapid planar mean flow measurements as well as observation of low-frequency phenomena.
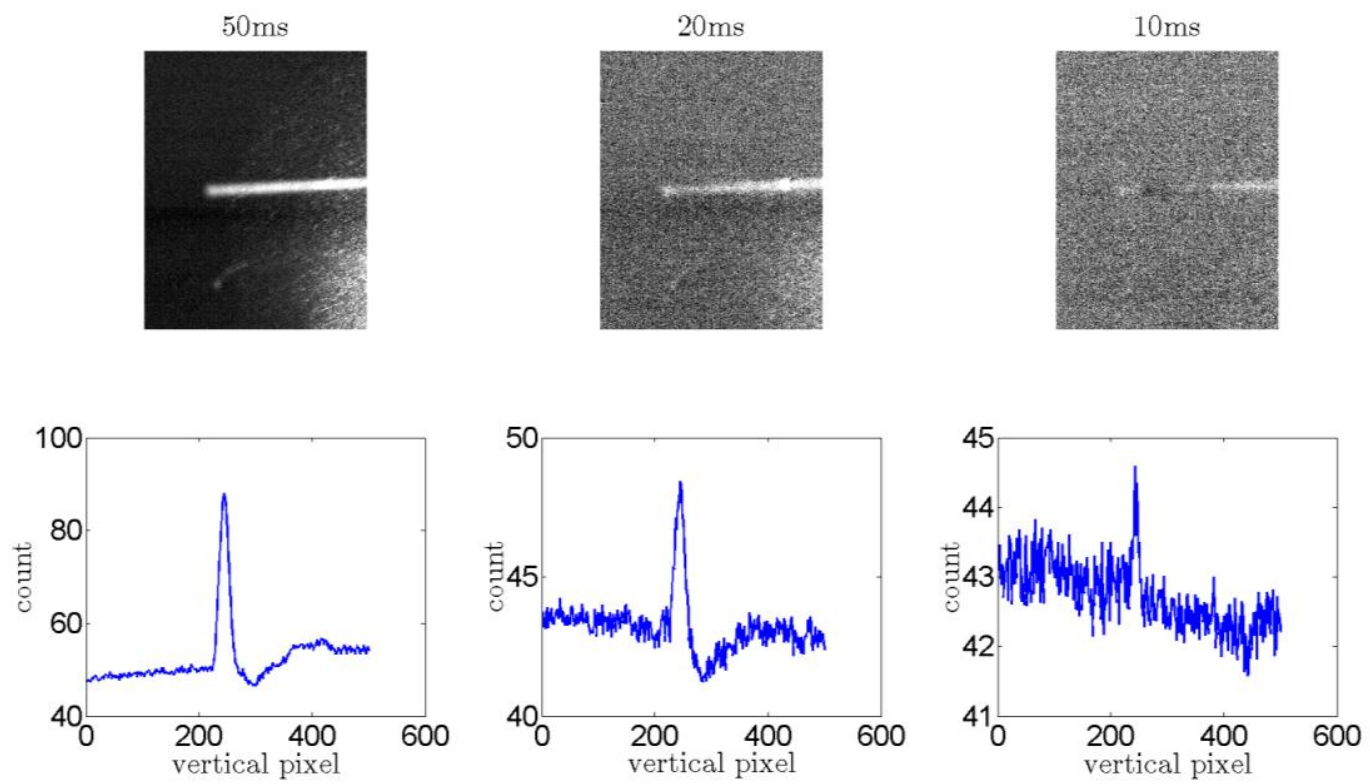

Figure 5. Imaging using a CW laser source at $2 \mathrm{~W}$ power. Left to right: $50 \mathrm{~ms}, 20 \mathrm{~ms}, 10 \mathrm{~ms}$ exposure. Top row: raw images rescaled to accentuate the fluorescence signal (laser beam is horizontal line). Bottom row: mean pixel counts horizontally averaged across the image. 


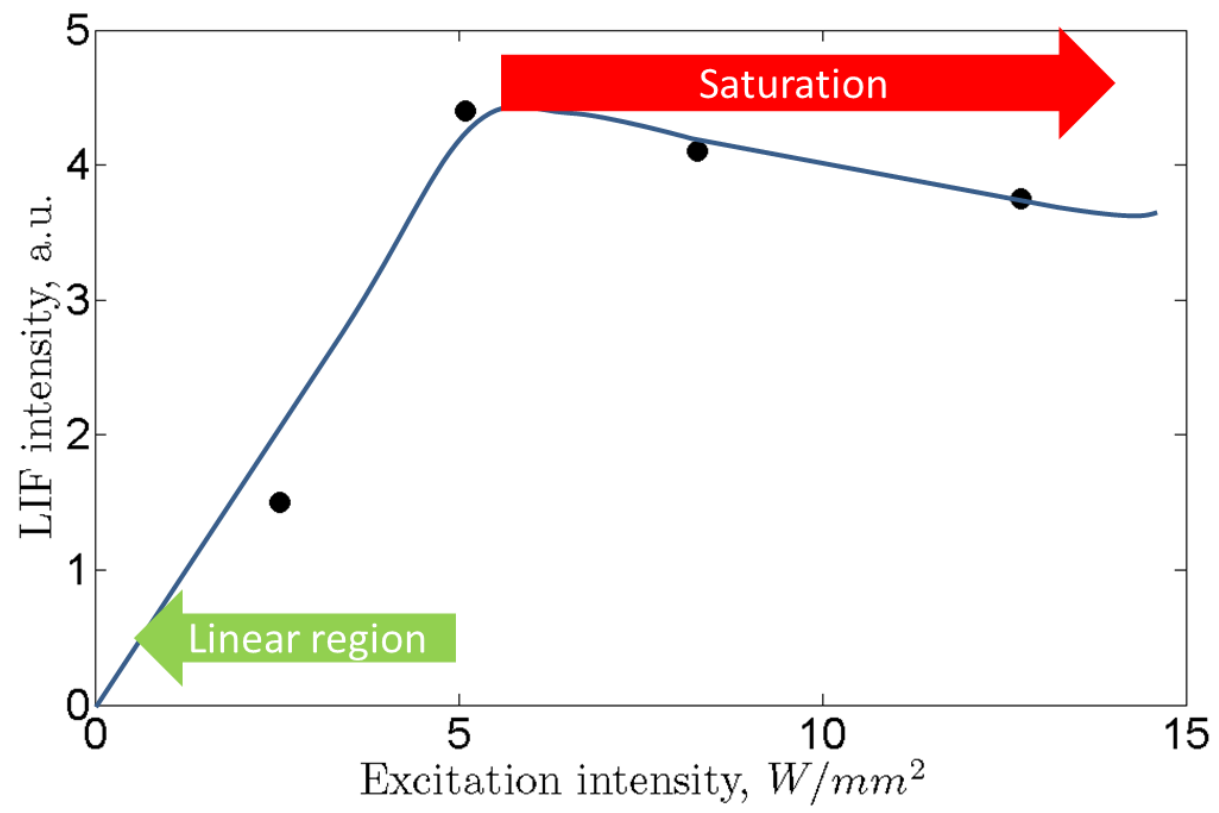

Figure 6. DCF Batch 58 doped particles power sensitivity. Symbols are actual data; curve is a notional curve drawn to illustrate the linear/saturation trend.

3. Relationship between Mie and LIF signals, 2-band LIF

The ratio of LIF signal to Mie signal was found to be non-uniform, varying considerably from particle to particle. In order to quantify the variation, we consider the standard deviation $(\sigma)$ of all burst samples for the LIF to Mie signal ratio $(\psi)$. The mean signal ratio within a single burst is computed via the burst time-series as

$$
\psi=\frac{\int_{\text {burst }} I_{L I F}}{\int_{\text {burst }} I_{\text {Mie }}}=\frac{\overline{I_{L I F}}}{\overline{I_{M i e}}}
$$

Where $\int_{\text {burst }}$ indicates time integration of a single particle burst. This parameter gives the ratio of the fluorescence yield to the Mie yield via integration of the voltage signal once photomultiplier gain is taken into account. Regarding this ratio, we note that while the Mie scattering is proportional to the square of particle diameter $\left(\sim \mathbf{D}^{2}\right.$ particle $)$, the LIF signal should scale proportionally to the volume of the particle $\left(\sim \mathbf{D}^{\mathbf{3}}\right.$ particle $)$, partially explaining some of the differences in yield exhibited from batch to batch; as indicated in Table 1, batch mean diameters were not constant. The dependence of the ratio $\psi$ on particle diameter would also indicate that the distribution of $\psi$ should behave similarly to the distribution in diameter. Consider the normalized standard deviation of $\psi$,

$$
\tilde{\psi}=\frac{\sigma(\psi)}{\bar{\psi}}
$$

where $\bar{\psi}$ is the ensemble mean of $\psi$ for all particles measured. It is expected from the dimensional analysis above that $\tilde{\psi} \propto \sigma\left(D_{\text {particle }}\right) / \overline{D_{\text {particle }}}$, and indeed, results from several batches characterized (including some not listed in Table 1) exhibit a strong correlation between diameter and $\psi$ statistics. This correlation is depicted in Fig. 7. The largest fluctuations for the LIF to Mie ratio were observed for the DCF batch 60 with a value of $\tilde{\psi}$ of about $82 \%$; from data in Table 1 , the value of $\sigma\left(D_{\text {particle }}\right) / \overline{D_{\text {particle }}}$ in the same batch is approximately $89 \%$. 
The limited correlation between the LIF and Mie signals is further exhibited in Figs. 8 and 9. The joint probability density in Fig. 8 (left) graphically illustrates that Mie intensity is a weak predictor of LIF intensity. For several flow temperatures measured over the range of $20-120^{\circ} \mathrm{C}$, the correlation coefficient between the Mie and LIF signal intensity is approximately 0.6 or less. It is clear that single shot measurements of fluorescencedependent scalar flow quantities such as temperature would not be possible using the $\psi$ parameter due to the strong particle diameter dependence of its value.

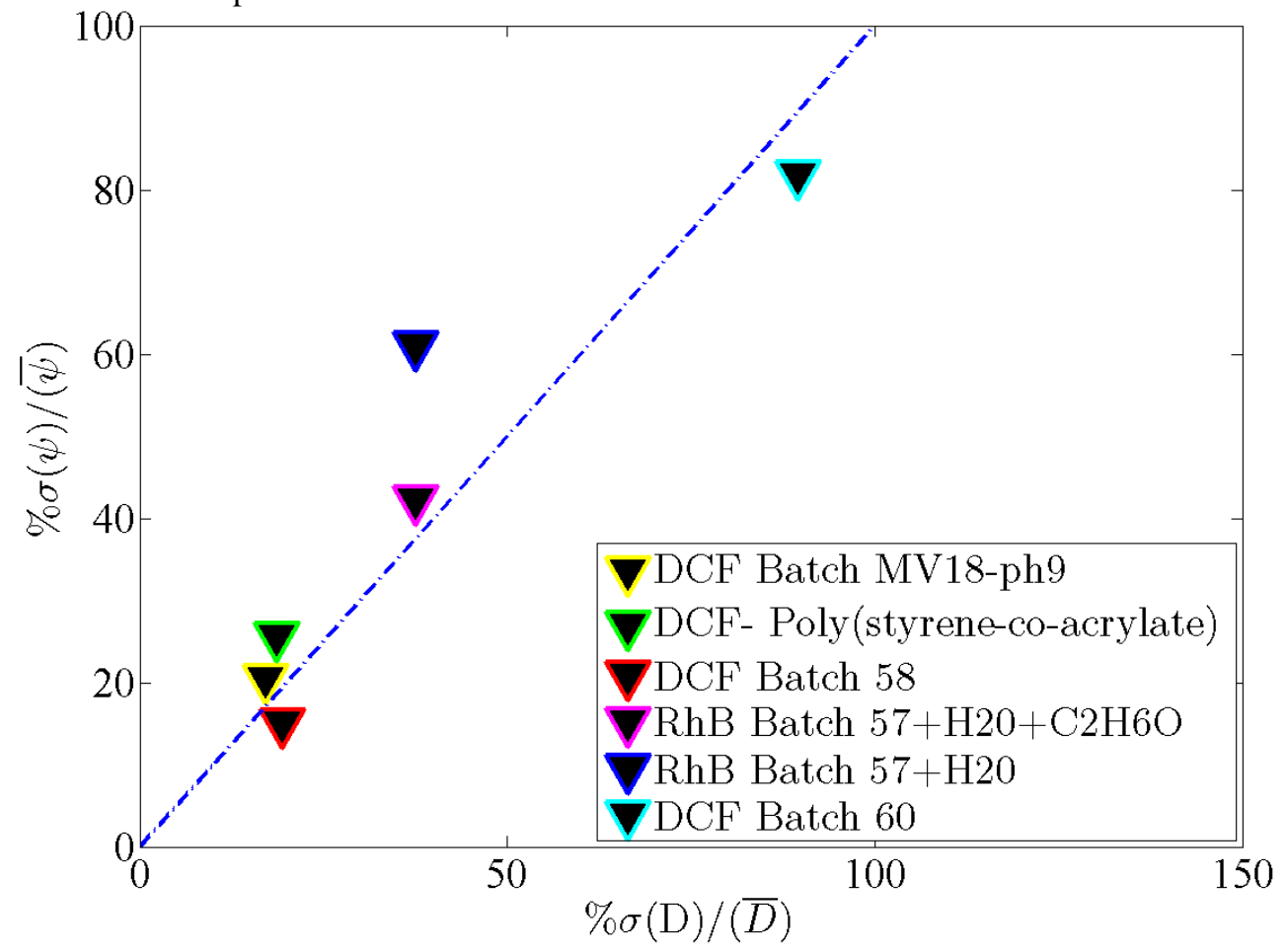

Figure 7. Relationship between the standard deviation in particle diameter (horizontal axis) and the standard deviation from single particle LIF-to-Mie fluorescence intensity measurements (vertical axis). RhB Batch 57 doped PSLs were tested both in distilled water solution and in water-ethanol solution. DCF batches MV18-ph9 and Poly(styrene-co-acrylate) were in the $\mathbf{2 0 - 2 5 \%}$ fluctuation range, while the lowest fluctuation value was found for Batch58 at $15 \%$.

The two-band LIF technique solves the issue with single-shot diameter dependence, along with other interferences such as excitation intensity and dye non-uniformity, exhibited by $\psi$. The joint probability map of normalized pass $(539 \mathrm{~nm}-559 \mathrm{~nm})$ and rejection $(>559 \mathrm{~nm})$ band LIF intensity (Fig. 8 right) shows strong correlations for single-shot measurements of DCF Batch 60 particles, in stark contrast to the LIF/Mie band comparison (left). The correlation coefficients between single-shot measurements were $>0.95$ for all flow temperatures less than $100{ }^{\circ} \mathrm{C}$, the temperature at which particles undergo glass transition and begin melting. Further, the ratio of the pass to rejection band LIF intensity,

$$
\eta \equiv \frac{\int_{\text {burst }} I_{\text {LIF Reject }}}{\int_{\text {burst }} I_{\mathrm{LIF} \text { Pass }}}=\frac{\overline{I_{\mathrm{LIF} \mathrm{Reject}}}}{\overline{I_{\mathrm{LIF} \text { Pass }}}}
$$

is distributed narrowly compared with all intensity measurement sets (Mie, pass or rejection band LIF); for example, see the histograms for DCF batch 60 in Fig. 10 and the intensity standard deviations of all three batches in Fig. 11. 

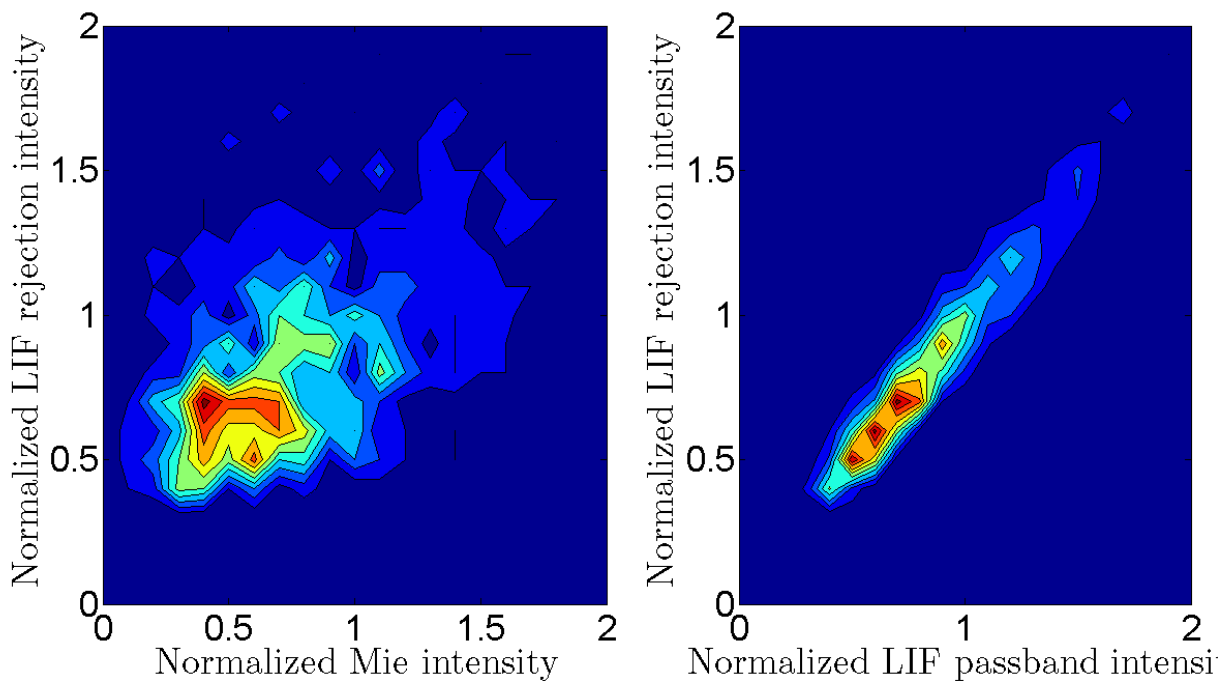

Normalized LIF passband intensity

Figure 8. Joint probability maps of single-shot measurements for DCF batch 60. Left: joint LIF rejection band and Mie intensity. Right: joint LIF pass and rejection bands.

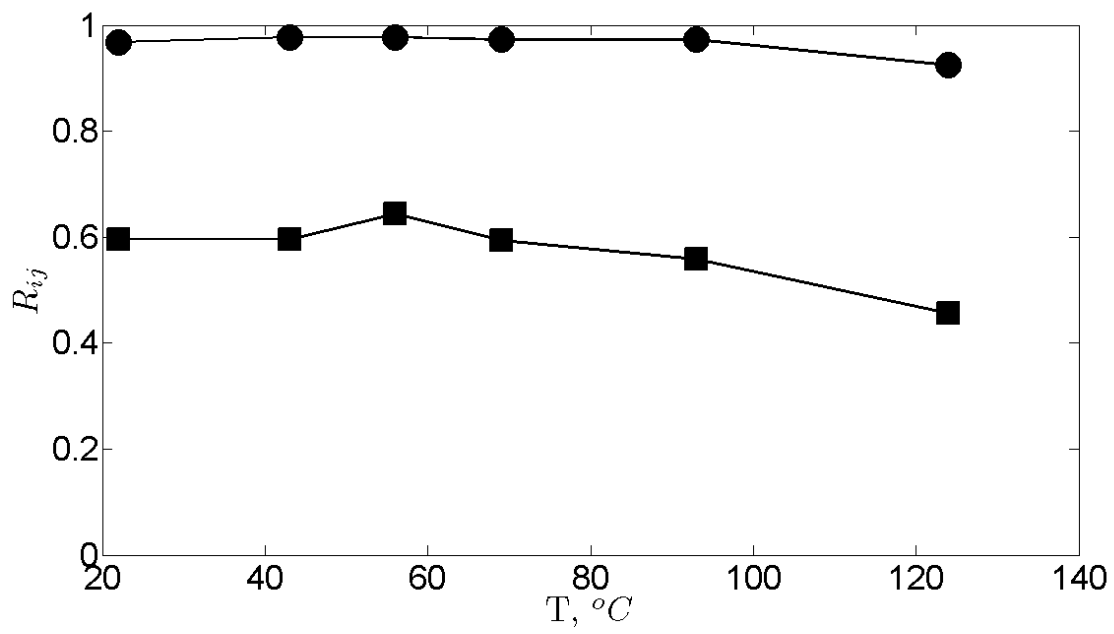

Figure 9. Correlation coefficients for single-shot burst intensity integration for the LIF rejection band/Mie band (squares) and pass/rejection LIF bands (circles) for DCF Batch 60. 


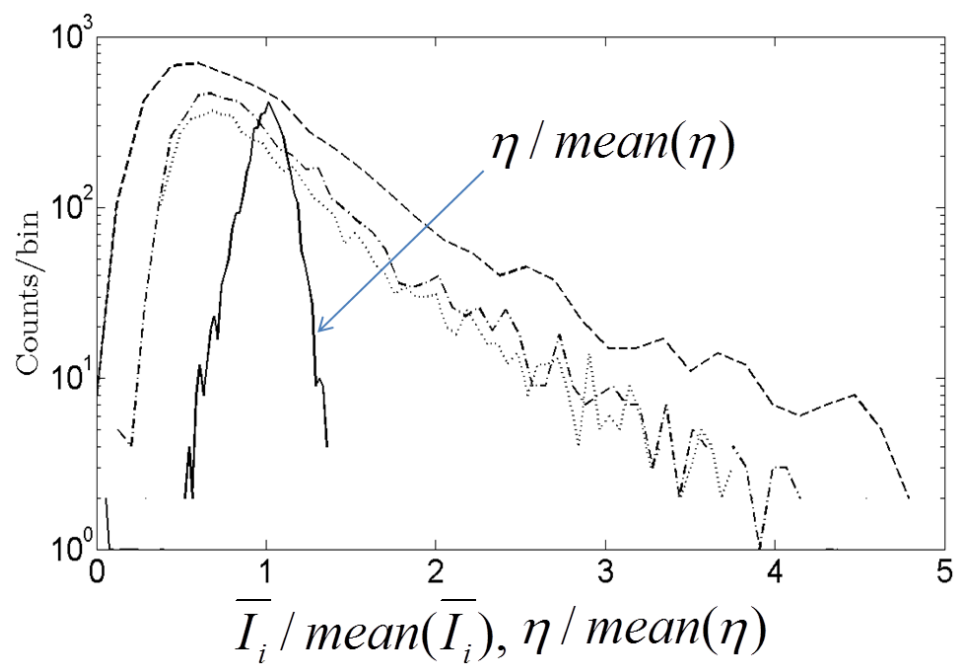

Figure 10. DCF batch 60: histograms for LIF pass (...), reflected (-.-), two-band LIF ratio $\eta$ (solid) and Mie (--) single-shot intensity measurements.

Statistics comparisons among the batches tested indicate that DCF batch 60 would perform strongest for twoband measurements (Figs. 11 and 12). The mean-normalized standard deviations for the LIF pass, rejection and ratio bands in Fig. 11 show a clear decrease in the standard deviation for the ratio compared with the individual signals. For the experimental setup, in which flow temperature variations were minimal, the expectation would be that variance in $\eta$ would represent a fundamental uncertainty in the measurement technique, such as noiselimited single-shot intensity estimation. This assertion was confirmed in the measurements.

As reported previously [2], particles doped with RhB exhibit very weak fluorescence in the chosen passband range (539 nm-559 nm), leading to low signal-to-noise ratio (SNR) for the passband and increased variance in the LIF ratio. Likewise, DCF batch 58 (red symbols in Fig. 11) exhibited generally weak fluorescence on both pass and rejection bands, leading to low SNR for both LIF signals and an increased variance in the LIF ratio. The DCF batch 60 particles (green symbols in Fig. 11) exhibited nearly equal signal on the pass and rejection bands, and the SNR for both was very good. The pass/rejection band correlation coefficients are graphed versus temperature in Fig. 12. They directly reflect the trends just discussed throughout the temperature range examined - uncorrelated content led to increased standard deviations in the values of $\eta$.
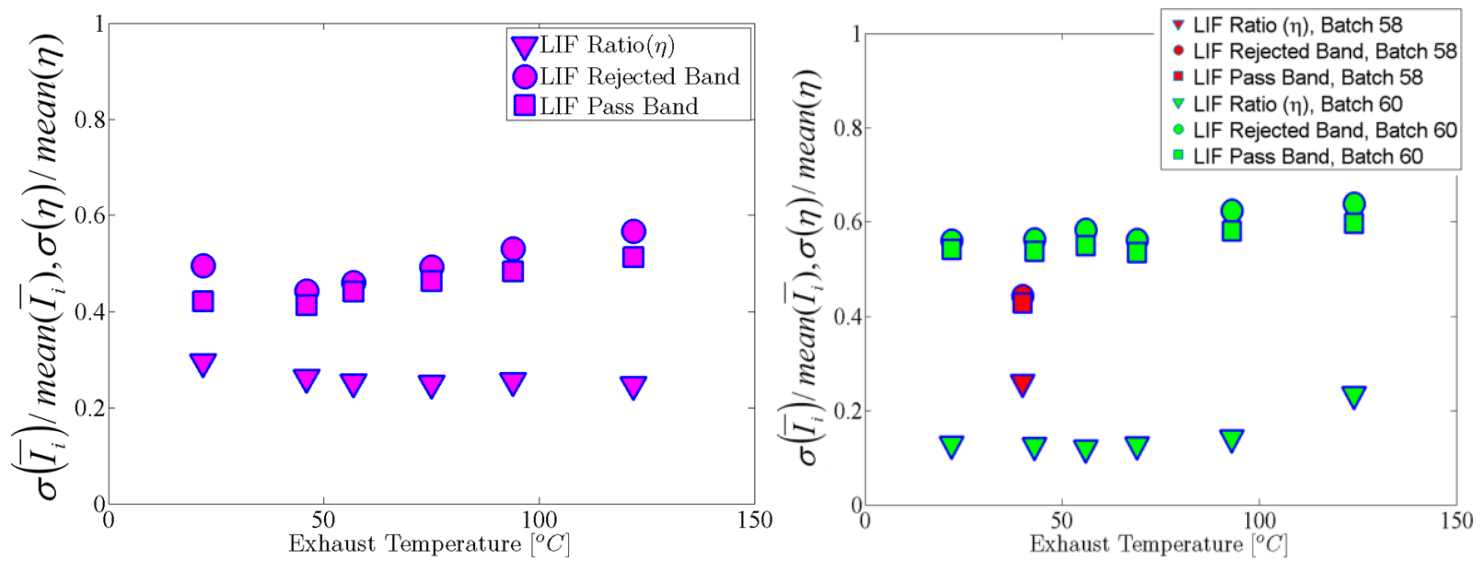

Figure 11. Normalized fluctuations of the LIF signals (Pass, Reflected and Band Ratio) for RhB and DCF doped particles. Left: RhB batch 57. Right: red symbols - DCF batch 58, green symbols - DCF batch 60. 


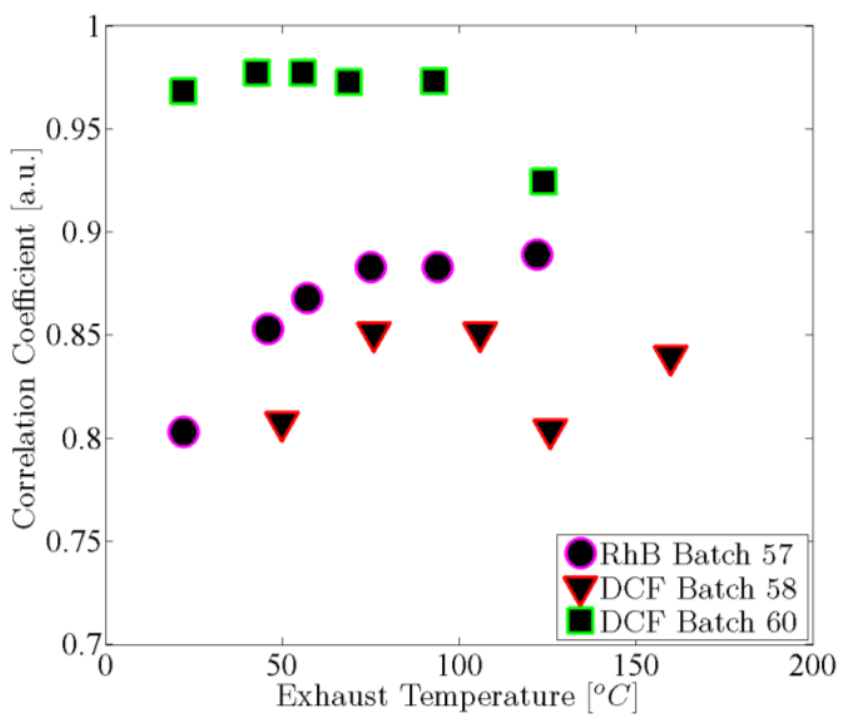

Figure 12. Correlation coefficients for LIF pass/rejection band signals versus flow temperature for RhB batch 57, DCF batches 58 and 60. The best correlation was found to be 0.977 for the DCF batch 60 around $50^{\circ} \mathrm{C}$.

\section{A note on single-shot temperature uncertainties}

The key result from Fig. 11, single-shot standard deviations on the order of $10 \%$ of the mean LIF pass/rejection band ratio were measured for DCF batch 60, will be considered further for estimating the performance of these particles for temperature measurements.

Given the temperature-dependent fluorescence yield of the DCF-doped particles shown in Figs. 3 and 4, one may formulate a simple model relating $\eta$ and temperature:

$$
\eta=A T^{n}
$$

where $A$ and $n$ are calibration constants for the dye-doped particles. Given measurements of $\eta$, the temperature may be determined by solution of equation (6) or a look-up of calibration data. The uncertainty in using equation (6) for a temperature measurement is

$$
(\delta T)^{2}= \pm\left[\left(\frac{\partial T}{\partial \eta} \delta \eta\right)^{2}+\left(\frac{\partial T}{\partial A} \delta A\right)^{2}+\left(\frac{\partial T}{\partial n} \delta n\right)^{2}\right]
$$

which will reduce to

$$
\delta T \approx \pm \frac{\partial T}{\partial \eta} \delta \eta
$$

when assessing single-shot uncertainty and assuming that $A$ and $n$ are invariant throughout a given particle batch.

Table 2 was constructed for a range of realistic temperature sensitivities $\left(\frac{\partial T}{\partial \eta}\right)$ and two-band ratio uncertainties $(\delta \eta)$. The case of $\partial T / \partial \eta=125^{\circ} \mathrm{C}$ is approximated from the slope in Fig. 4. If $\delta \eta=0.1(10 \%)$, then the anticipated temperature precision is $\pm 12.5^{\circ} \mathrm{C}$. With higher signal-to-noise ratios, it may be possible to reduce $\delta \eta$ by a factor of 2 or 4 , yielding single-digit temperature precisions. Alternatively, for the same $\delta \eta$ demonstrated herein, a steeper slope of the $\eta(T)$ curve of say $66{ }^{\circ} \mathrm{C}$ would achieve single-digit precision. As efforts progress, it is expected that improvements in both parameters are feasible, with the prospect of sub-degree precision. 
Table 2. Single-shot measurement temperature uncertainties ( $\delta \mathrm{T}$, in ${ }^{\circ} \mathrm{C}$, unshaded boxes) computed from equation (8) for representative sensitivity and normalized signal variance values.

\begin{tabular}{|c|c|c|c|c|c|}
\hline$\delta \eta$ & $\frac{\partial T}{\partial \eta}=125^{\circ} \mathrm{C}$ & $\frac{\partial T}{\partial \eta}=66^{\circ} \mathrm{C}$ & $\frac{\partial T}{\partial \eta}=33^{\circ} \mathrm{C}$ & $\frac{\partial T}{\partial \eta}=15{ }^{\circ} \mathrm{C}$ & $\frac{\partial T}{\partial \eta}=7{ }^{\circ} \mathrm{C}$ \\
\hline 0.025 & 3.1 & 1.7 & 0.8 & 0.4 & 0.2 \\
\hline 0.05 & 6.3 & 3.3 & 1.7 & 0.8 & 0.4 \\
\hline 0.1 & 12.5 & 6.6 & 3.3 & 1.5 & 0.7 \\
\hline 0.2 & 25.0 & 13.2 & 6.6 & 3.0 & 1.4 \\
\hline
\end{tabular}

\section{Conclusion}

Polystyrene latex spheres have been doped with fluorescent dyes and characterized using LIF and Miescattering signals from single particles. Previously published results indicate that signal strengths are sufficient for obtaining velocimetry results from the LIF signal using an LDV optical arrangement. The relative signal strength of fluorescence and Mie signals from Rhodamine B and DCF-doped PSLs were measured quantitatively using PMTs, and the LIF yield as a ratio of Mie scattering showed a strong correlation with particle diameter variance, as predicted from dimensional arguments for LIF and Mie scattering. Imaging was demonstrated using $\mathrm{CW}$ lasers at exposure times of $10 \mathrm{~ms}$ for an excitation power of $2 \mathrm{~W}$. A significant consequence for imaging applications was that LIF saturation occurred at a level too low to produce sufficient signal using pulsed lasers. Despite this, the single-shot results using PMT detectors indicate that particle imaging with CW excitation should be feasible for integration times on the order of $10 \mathrm{~ms}$ with an intensified camera, and integration times on the order of $10 \mathrm{~ms}$ may be used for unintensified cameras. Such imaging could potentially be useful, for example, in time-averaged concentration measurements in two-stream experiments (mixing layers) in conjunction with velocity obtained from LDV. The study conducted for the two-band LIF characterization indicates that DCF batch 60, a formulation with increased $\mathrm{pH}$, exhibits high correlation coefficients $(>0.95)$ over large temperature ranges, demonstrating the feasibility of using two-band LIF given a dye with sufficient temperature sensitivity. Finally, an estimation of expected uncertainties for the technique indicates that the current developments may produce temperature precision to $\pm 12.5^{\circ} \mathrm{C}$, while it is feasible that on-going efforts to improve dye temperature sensitivity and measurement signal-to-noise ratio may result in sub-degree precision.

\section{Acknowledgments}

The authors acknowledge the support of the NASA ARMD Seedling Fund and NIA Cooperative Agreement NNL09AA00A.

\section{References}

1. Tiemsin, P., and Wohl, C. "Refined Synthesis and Characterization of Controlled Diameter, Narrow Size Distribution Microparticles for Aerospace Research Applications," NASA Technical Memorandum, 2012, pp. TM-2012-217591.

2. Lowe, K. T., Maisto, P., Byun, G., Simpson, R. L., Verkamp, M., Danehy, P. M., Tiemsin, P. I., and Wohl, C. J. "Laser velocimetry with fluorescent dye-doped polystyrene microspheres," Optics Letters Vol. 38, No. 8, 2013, pp. 1197-1199.

doi: 10.1364/OL.38.001197

3. Stevenson, W. H., dos Santos, R., and Mettler, S. C. "A laser velocimeter utilizing laser-induced fluorescence," Applied Physics Letters Vol. 27, No. 7, 1975, pp. 395-6.

doi: $10.1063 / 1.88506$

4. Dunand, P., Castanet, G., and Lemoine, F. "A two-color planar LIF technique to map the temperature of droplets impinging onto a heated wall," Experiments in Fluids Vol. 52, No. 4, 2012, pp. 843-856.

doi: 10.1007/s00348-011-1131-1

5. Sutton, J., Fisher, B., and Fleming, J. "A laser-induced fluorescence measurement for aqueous fluid flows with improved temperature sensitivity," Experiments in Fluids Vol. 45, No. 5, 2008, pp. 869-81.

doi: 10.1007/s00348-008-0506-4

6. Brubach, J., Patt, A., and Dreizler, A. "Spray thermometry using thermographic phosphors," Applied Physics B: Lasers and

Optics Vol. 83, No. 4, 2006, pp. 499-502.

doi: $10.1007 / \mathrm{s} 00340-006-2244-8$ 
7. Someya, S., Li, Y., Ishii, K., and Okamoto, K. "Combined two-dimensional velocity and temperature measurements of natural convection using a high-speed camera and temperature-sensitive particles," Experiments in Fluids Vol. 50, No. 1, 2011, pp. 65-73.

doi: 10.1007/s00348-010-0894-0

8. Benner, R. E., Barber, P. W., Owen, J. F., and Chang, R. K. "Observation of structure resonances in the fluorescence spectra from microspheres," Physical Review Letters Vol. 44, No. 7, 1980, pp. 475-8.

doi: 10.1103/PhysRevLett.44.475

9. Hochareon, P., Manning, K. B., Fontaine, A. A., Tarbell, J. M., and Deutsch, S. "Wall shear-rate estimation within the 50cc Penn State artificial heart using particle image velocimetry," Transactions of the ASME. Journal of Biomechanical Engineering Vol. 126, No. 4, 2004, pp. 430-7.

doi: $10.1115 / 1.1784477$

10. Dong, R., Chu, S., and Katz, J. "Quantitative visualization of the flow within the volute of a centrifugal pump. Part A: technique," Journal of Fluids Engineering, Transactions of the ASME Vol. 114, No. 3, 1992, pp. 390-395. 